\section{()} OPEN ACCESS

\title{
GRANADA consensus on analytical approaches to assess associations with accelerometer-determined physical behaviours (physical activity, sedentary behaviour and sleep) in epidemiological studies
}

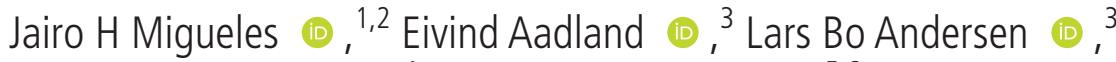

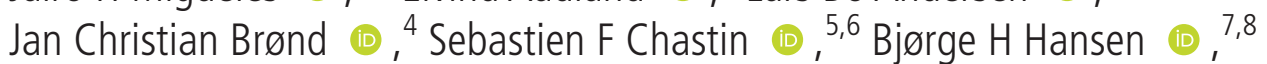

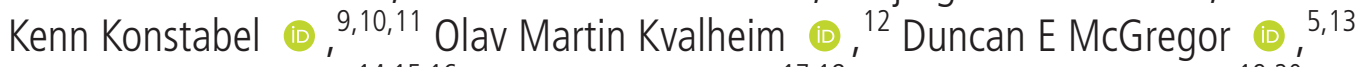 \\ Alex V Rowlands (1) ${ }^{14,15,16}$ Séverine Sabia (1) ${ }^{17,18}$ Vincent T van Hees (1) , 19,20 \\ Rosemary Walmsley $\mathbb{C}^{2},{ }^{21,22}$ Francisco B Ortega 다, 1,23 External review group
}

\begin{abstract}
- Additional supplemental material is published online only. To view, please visit the journal online (http://dx.doi. org/10.1136/bjsports-2020 103604).
\end{abstract}

For numbered affiliations see end of article.

Correspondence to Jairo $\mathrm{H}$ Migueles and $\mathrm{Dr}$ Francisco B Ortega, Department of physical education and sport, University of Granada, 18071 Granada, Spain:

jairohm@ugr.es, ortegaf@ugr.es

Accepted 20 March 2021
Check for updates

(C) Author(s) (or their employer(s)) 2021. Re-use permitted under CC BY-NC. No commercial re-use. See rights and permissions. Published by BMJ.

To cite: Migueles JH, Aadland E, Andersen LB, et al. Br J Sports Med Epub ahead of print: [please include Day Month Year]. doi:10.1136/ bjsports-2020-103604

\section{ABSTRACT}

The inter-relationship between physical activity, sedentary behaviour and sleep (collectively defined as physical behaviours) is of interest to researchers from different fields. Each of these physical behaviours has been investigated in epidemiological studies, yet their codependency and interactions need to be further explored and accounted for in data analysis. Modern accelerometers capture continuous movement through the day, which presents the challenge of how to best use the richness of these data. In recent years, analytical approaches first applied in other scientific fields have been applied to physical behaviour epidemiology (eg, isotemporal substitution models, compositional data analysis, multivariate pattern analysis, functional data analysis and machine learning). A comprehensive description, discussion, and consensus on the strengths and limitations of these analytical approaches will help researchers decide which approach to use in different situations. In this context, a scientific workshop and meeting were held in Granada to discuss: (1) analytical approaches currently used in the scientific literature on physical behaviour, highlighting strengths and limitations, providing practical recommendations on their use and including a decision tree for assisting researchers' decision-making; and (2) current gaps and future research directions around the analysis and use of accelerometer data. Advances in analytical approaches to accelerometer-determined physical behaviours in epidemiological studies are expected to influence the interpretation of current and future evidence, and ultimately impact on future physical behaviour guidelines.

\section{INTRODUCTION}

Physical activity (PA), sedentary behaviour (SB) and sleep, collectively described as physical behaviours, ${ }^{1}$ are of interest to many researchers from previously separate fields. Accounting for the inter-relations of these behaviours is important because: (1) they share the 24 hours of the day (ie, closure), so change in one behaviour results in change in others; and (2) the relation of a specific behaviour with health depends on other behaviours (eg, SB and mortality relation depends on moderate-to-vigorous PA
$\left.(\mathrm{MVPA})^{23}\right)$. These inter-relations should be considered in modelling their association with health, with attention to collinearity issues potentially leading to spurious findings.

Accelerometers are increasingly being used to estimate different constructs/dimensions of physical behaviours (eg, types (walking, cycling, dancing), intensities (light, moderate, vigorous), and postures (reclining, sitting, standing)). Other constructs focus more on the description of the acceleration signal (eg, time spent within acceleration bands with no energy expenditure interpretation (eg, $\mathrm{min} /$ day between 0 and $100 \mathrm{mg}$ ), or the acceleration above which the most active $30 \mathrm{~min}$ of the day occur). The data-analytical approach usually includes: (1) reduction of the acceleration signal into meaningful behaviours/descriptors; (2) mathematical treatment of the descriptors if needed and (3) selection of the statistical model. Multiple choices are available for each step, and decisions should be adapted to the research question and account for potential collinearity issues arising from behaviours' inter-relationships. However, there are currently no consensus/recommendations to help to choose the most appropriate approach. Online supplemental appendix 1 presents the different choices for descriptors, mathematical treatments and statistical models discussed in the Analytical approaches section.

The 'International Workshop: A focus on statistical methods to analyse accelerometer-measured PA' was held in Granada on 21-22 October 2019. This event brought together a panel of researchers to discuss, reach consensus, and provide recommendations about the most frequently used analytical approaches in the field and about future research directions in physical behaviour epidemiology. The focus was on modelling physical behaviour constructs (mainly related to PA and SB, although we also included sleep to cover the 24-hour continuum) as exposure variables and health indicators as outcomes. We covered time-use descriptors as those quantified in time over the day, and acceleration-based as those quantified as acceleration magnitude. 
Table 1 Description of accelerometer-based descriptors of physical behaviours

\begin{tabular}{lll}
\hline Descriptor & Brief description & Examples \\
\hline $\begin{array}{l}\text { Average acceleration or steps } \\
\text { per day }\end{array}$ & Arithmetic average of the processed acceleration throughout the measurement period or per day. & $293646-48$ \\
\hline Time-use behaviours & $\begin{array}{l}\text { Estimates of time spent in physical activity intensities (eg, LPA, MPA, VPA), types (eg, walking, running, cycling), or SB, optionally } \\
\text { expressed in bouted and unbouted behaviour. These estimates can be derived with heuristic methods or ML. }\end{array}$ & $2949-52$ \\
\hline $\begin{array}{l}\text { The intensity spectrum is an extension of cut-points which attempts to provide a much more detailed description of the physical } \\
\text { activity intensity pattern. Instead of using cut-points representative of SB, LPA, MPA or VPA, the cut-points are arbitrarily selected } \\
\text { to obtain a wider range of intensity bands. }\end{array}$ & 323353 \\
\hline Intensity gradient & $\begin{array}{l}\text { The intensity gradient describes the negative curvilinear relationship between physical activity intensity and the time accumulated } \\
\text { at that intensity during the 24-hour day. }\end{array}$ & 3646 \\
\hline MX metrics & $\begin{array}{l}\text { The acceleration above which a person's most active X minutes/time (MX) are accumulated, to focus on a person's most active } \\
\text { periods of the day. }\end{array}$ & 5455 \\
\hline Acceleration functions & $\begin{array}{l}\text { Description of the accelerometer data with a function rather than with a scalar. Functions seek a more detailed description of the } \\
\text { accelerometer data without making a priori assumptions. }\end{array}$ & 383956 \\
\hline Other indicators & $\begin{array}{l}\text { Apart from the descriptors related to energy intensity or acceleration levels, an array of metrics can provide complementary } \\
\text { information, such as: physical activity domain, circadian rhythmicity, timing, sleep efficiency, etc. }\end{array}$ & 345758 \\
\hline LPA, light physical activity; ML, machine learning; MPA, moderate physical activity; SB, sedentary behaviour; VPA, vigorous physical activity.
\end{tabular}

Data collection decisions are outside our focus, although decisions on body attachment site, ${ }^{4-8}$ number of days recorded, ${ }^{9}$ treatment of weekdays and weekend days, ${ }^{10}{ }^{11}$ seasonality, ${ }^{12}$ among others, affect the ability of accelerometer data to identify specific constructs/aspects of physical behaviours. For example, attaching the accelerometer to the hip, wrist or thigh may be considered depending on the constructs of interest (eg, PA intensity, ${ }^{13-15}$ postures ${ }^{16}$ or sleep patterns, ${ }^{1718}$ among others). A recent consensus report discussed best practices on these decisions. ${ }^{19}$

\section{ANALYTICAL APPROACHES: DISCUSSION AND PRACTICAL CONSIDERATIONS}

This section discusses different analytical approaches' applicability in various situations (or research questions). Analytical approaches include the combination of accelerometer descriptors (table 1, online supplemental appendix 1 (Section 1)) and statistical models (table 2, online supplemental appendix 1 (Section 3)) with and without mathematical (compositional) transformation (online supplemental appendix 1 (Section 2)). We provide practical considerations on (1) informativeness of each analytical approach for public health messaging and (2) appropriateness of the analytical approaches for certain research questions. Additionally, table 3 shows the performance of these approaches regarding closure or collinearity, relationship assumptions and interpretation for PA guidelines.

\section{Total PA and linear regression}

Average acceleration (or steps per day) provides the simplest estimate of the overall movement and proxy for total daily PA-related energy expenditure. Statistical interpretation of findings using linear regression is straightforward since there is a single variable representing the overall activity volume. Thus, codependence with other explanatory variables is not usually a concern and linear regression models are an option for the analysis. The opinion of the consensus group is that the average acceleration is useful for reducing the confounding effect of PA in a given association analysis (eg, is the association of sugar consumption with body mass index dependent on overall PA?), or as the main exposure in cases where it explains a large proportion of the PA-related energy expenditure in a certain cohort (eg, is PA-related energy expenditure associated with protein intake?). Beyond this, the average acceleration alone is not very informative relative to associations of specific physical behaviours with health outcomes, limiting its applicability for public health messaging. A recent study proposed the minimum clinically informative difference for average acceleration from wrist data, ${ }^{20}$ but further studies are needed. Although these descriptors cannot be interpreted in terms of meeting or not meeting the PA guidelines, they may be the best descriptor to test the 'move more' message reported in several guidelines.

Table 2 Brief description of approaches to analyse associations between physical behaviours and health outcomes

\begin{tabular}{|c|c|c|}
\hline Statistical model & Brief description & Examples \\
\hline $\begin{array}{l}\text { Linear regression } \\
\text { modelling }\end{array}$ & $\begin{array}{l}\text { Traditional models establishing the relationship between a set of explanatory variables and an outcome (ie, health outcome). Exposure is } \\
\text { usually limited to a single time-use behaviour. Interpretation is in terms of increasing time in one behaviour. }\end{array}$ & 5960 \\
\hline $\begin{array}{l}\text { Isotemporal substitution } \\
\text { model }\end{array}$ & $\begin{array}{l}\text { Isotemporal substitution models examine the theoretical effects of displacing a fixed duration of time between behaviours. Given the fixed } \\
\text { and finite duration of a day, increasing time in one movement behaviour (eg, LPA) will result in a net equal and opposite change in other } \\
\text { movement behaviours (eg, SB). Interpretation is in terms of substituting one behaviour for other behaviours. }\end{array}$ & 6162 \\
\hline $\begin{array}{l}\text { Multivariate pattern } \\
\text { analysis }\end{array}$ & $\begin{array}{l}\text { A regression approach/analysis that can handle an unlimited number of multicollinear explanatory variables by using latent variable } \\
\text { modelling. Models are cross-validated to optimise predictive ability. Interpretation is based on the complete pattern of associations among } \\
\text { the explanatory variables in relation to the outcome. }\end{array}$ & $2563-66$ \\
\hline Functional data analysis & $\begin{array}{l}\text { Functional data analysis is an extension of scalar regression where the exposure or outcome is defined as a function rather than a scalar } \\
\text { variable. The function can describe the full distribution of intensity of acceleration or the time-series of acceleration over the day. The } \\
\text { function can be included in linear regression analysis through dimensional reduction techniques. Interpretation is in terms of certain } \\
\text { accelerometer trace shapes. }\end{array}$ & $373867-69$ \\
\hline Machine learning (ML) & $\begin{array}{l}\text { ML entails a broad range of techniques to automate the learning of high-dimensional and/or non-linear patterns in data with predictive } \\
\text { ability (supervised } \mathrm{ML} \text { ) or data reduction (unsupervised } \mathrm{ML} \text { ) as its core priority. }\end{array}$ & 417071 \\
\hline
\end{tabular}


Table 3 Summary of analytical approaches' (including descriptor, mathematical transformation and statistical model) strengths and limitations in relation to closure, collinearity, relation-shape assumptions and interpretation relative to public health guidelines

\begin{tabular}{|c|c|c|c|c|c|c|c|c|c|}
\hline Descriptor & $\begin{array}{l}\text { CoDA } \\
\text { transform }\end{array}$ & $\begin{array}{l}\text { Statistical } \\
\text { modelling }\end{array}$ & $\begin{array}{l}\text { Risk of } \\
\text { closure?* }\end{array}$ & $\begin{array}{l}\text { Risk of } \\
\text { collinearity? }\end{array}$ & $\begin{array}{l}\text { Handles } \\
\text { closure? }\end{array}$ & $\begin{array}{l}\text { Handles } \\
\text { collinearity? }\end{array}$ & $\begin{array}{l}\text { Relationship } \\
\text { assumptions }\end{array}$ & $\begin{array}{l}\text { Allow investigation } \\
\text { of longitudinal } \\
\text { associations (eg, Cox } \\
\text { regression) }\end{array}$ & $\begin{array}{l}\text { Interpretation } \\
\text { relative to } \\
\text { guidelines? (eg, } \\
150 \mathrm{~min} / \text { week of } \\
\text { MVPA) }\end{array}$ \\
\hline $\begin{array}{l}\text { Average } \\
\text { acceleration }\end{array}$ & No & Linear & No & No & NA & NA & Linear & Yes & No \\
\hline \multirow{5}{*}{$\begin{array}{l}\text { Time-use } \\
\text { descriptors }\end{array}$} & No & Linear & Yes & Yes & No & No & Linear & Yes & Yes \\
\hline & Yes & Linear & Yes & Yes & Yes & In part $\dagger$ & Log-linear & Yes & Yes \\
\hline & No & ISO & Yes & Yes & Yes & No & Linear & Yes & Yes \\
\hline & No & MPA & Yes & Yes & No & No & Linear & Not at the moment & Yes \\
\hline & Yes & MPA & Yes & Yes & Yes & Yes & Log-linear & Not at the moment & Yes \\
\hline \multirow{5}{*}{$\begin{array}{l}\text { Intensity } \\
\text { spectrum }\end{array}$} & No & Linear & Yes & Yes & No & No & Linear & Yes & Yesł \\
\hline & Yes & Linear & Yes & Yes & Yes & In part $†$ & Log-linear & Yes & Yesł \\
\hline & No & ISO & Yes & Yes & Yes & No & Linear & Yes & Yesł \\
\hline & No & MPA & Yes & Yes & No & No & Linear & Not at the moment & Yesł \\
\hline & Yes & MPA & Yes & Yes & Yes & Yes & Log-linear & Not at the moment & Yesł \\
\hline \multirow[t]{2}{*}{ Intensity gradient } & No & Linear & No & No & NA & NA & Linear & Yes & No \\
\hline & No & FDA & No & No & NA & NA & $\begin{array}{l}\text { Fewer assumptions than } \\
\text { other models }\end{array}$ & Yes & Yes§ \\
\hline \multirow[t]{2}{*}{$\mathrm{MX}$ metrics } & No & Linear & Yes & Yes & No & No & Linear & Yes & Yesł \\
\hline & No & MPA & Yes & Yes & No & Yes & Linear & Not at the moment & Yesł \\
\hline $\begin{array}{l}\text { Other } \\
\text { acceleration } \\
\text { functions }\end{array}$ & No & FDA & No & No & NA & NA & $\begin{array}{l}\text { Fewer assumptions than } \\
\text { other models }\end{array}$ & Yes & Yes§ \\
\hline
\end{tabular}

${ }^{*}$ Closure refers to whether a certain descriptor is a specific part of the daily time constraint (ie, it is measured in time per day).

tIndicates that it solves the collinearity due to the closure, but collinearity can still exist across the CoDA-transformed variables.

FIndicates that the interpretation is made through a post-hoc application of validated cut-points to identify the PA intensity (eg, MVPA).

§Indicates that more work is needed on the interpretation of functional data analysis, an example can be found elsewhere. ${ }^{39}$

CoDA, compositional data analysis; FDA, functional data analysis; ISO, isotemporal substitution models; MPA, multivariate pattern analysis; MVPA, moderate-to-vigorous PA; MX, acceleration above which a person's most active $\mathrm{X}$ minutes/time are spent; $\mathrm{NA}$, not applicable; PA, physical activity.

\section{Time-use behaviours or intensity spectrum and linear regression}

Among time-use constructs, time spent in PA intensities is the most frequently used, while PA types and postures have gained momentum recently. These descriptors are often introduced in linear regression models to test the association of time spent in a certain intensity/behaviour with health outcomes. As it is widely used, it is useful for comparing estimates with other cohorts. The intensity spectrum is an extension of PA intensities with higher resolution energy bands. When using such time-use behaviours, requirements for bouts in these behaviours should be considered. We observe a lack of consensus in the literature on how a bout should be calculated, including the definition of both acceptable allowance drop period without terminating the bout and minimum and maximum duration. Bouts of $30 \mathrm{~min}$ for SB and $10 \mathrm{~min}$ for MVPA, often allowing short time intervals outside the behaviour of interest, are frequently used. ${ }^{21}$ It is unclear how much these choices are driven by a desire for harmonisation, by public health guidelines, or by evidence. Although observational data based on 1-minute or longer epoch lengths suggest that any bout duration can produce health benefits, randomised controlled trials investigating the effects of differing bout durations on health outcomes are lacking. ${ }^{21}$ Based on the observational studies, the recommendation about accumulating PA in certain bout durations has been excluded from recent guidelines. ${ }^{22}$

Time-use behaviours (or intensity spectrum) include a set of codependent variables, and linear regression does not handle closure and collinearity among explanatory variables. When using these descriptors, linear regression adjusted for all physical behaviour components may be affected by multicollinearity. ${ }^{23}$ Variance inflation factors are unable to explain inconsistencies between linear regression models sequentially excluding a behaviour from the explanatory variables ${ }^{24}$ and might not be an acceptable diagnostic indicator for the interdependency between time-use descriptors. ${ }^{24}$ Additionally, the assumption of linearity of the association between these descriptors and health outcome might not be sufficiently met for analyses to yield valid results. This consensus group recommends moving towards other analytical approaches more suitable for studying the codependencies among time-use behaviours. In this regard, transforming time-use behaviours using the compositional data transformation (isometric log ratio (ILR), online supplemental appendix 1 (Section 2)) represents an option. Using the ILR transformation, each variable indicates the time spent in a given behaviour relative to the time spent in the rest of behaviours of the composition (eg, SB, light PA, MVPA and sleep). In other words, it quantifies the effect of increasing the time in a behaviour while proportionally reducing the time in the rest. Pair-wise reallocations of time can be interpreted from linear regression predictions on specific time compositions arising from hypothetical reallocations of time rather than from regression coefficients (as in isotemporal substitution models, online supplemental appendix 1 (Section 3.2)). By transforming the variables, the codependency among the time-use descriptors relative to their time closure is solved (ie, it accounts for the codependency of time among variables). However, transformed variables can still be collinear, and collinearity should be investigated because linear regression cannot handle collinearity, regardless of its source. This is especially problematic when analysing the intensity spectrum since it provides a wide range of variables (usually more than 10) that are highly correlated, even if using ILR-transformed variables. ${ }^{25}$ As such, we recommend testing the correlations and risk of collinearity among the explanatory variables (even when compositionally transformed). In absence of high correlations and collinearity, linear regression can be appropriate. 
The opinion of the consensus group is that physical behaviour epidemiology should move to studying the combined effects and interactions of physical behaviours on health, and a feasible option is using ILR-transformed time-use descriptors and linear regression. ${ }^{24} 26$ This approach is informative for public health messaging as it provides information on combinations of behaviours (considering every behaviour that occurs in the day) which are beneficial for health. Clustering groups of people based on their behaviours is also an alternative to investigating the interactions between behaviours, although compositional analyses allow the variables to be studied on a continuous scale. With the intensity spectrum, the use of linear regression models is not possible because of collinearity issues in the variables (either transformed or not). ${ }^{25}$ The collinearity problem, however, can be solved by using partial least square (PLS) regression. Regression models can be used in different study designs, including longitudinal studies, either with absolute ${ }^{27-29}$ or compositional data. ${ }^{30}$ Linear regression with compositional data may need appropriate graphical representation of the results to interpret the magnitude of the association. ${ }^{24}$

\section{Time-use behaviours or intensity spectrum and isotemporal substitution models}

Isotemporal substitution modelling carries forward the main limitations of linear regression, that is, multicollinearity and assumption of linearity (as the magnitude of the association is derived from regression coefficients). These important limitations preclude us from recommending the use of isotemporal models with time-use descriptors. However, it is notable that this approach provides broadly similar findings to compositional ILR transformation of time-use descriptors and linear regression. ${ }^{31}$ Public health messaging can be complemented with information on the effect of reallocating daily time across behaviours (either with isotemporal substitution models or with linear regression with compositional data, the Time-use behaviours or intensity spectrum and linear regression section). The intensity spectrum has not been analysed with isotemporal substitution models thus far. We do not recommend such an analysis since the large number of variables in the intensity spectrum would complicate the interpretation.

\section{Time-use behaviours or intensity spectrum and multivariate pattern analysis}

Multivariate pattern analysis fully handles the collinearity among explanatory variables using latent variable modelling. Collinearity is approached as a dimensionality reduction problem in which the variance of the explanatory variables shared with the outcome is retained. Multivariate pattern analysis describes the pattern of associations for the descriptors with the outcome, accounting for the correlated structure of the data. Associations with health are interpreted for each descriptor (each PA intensity or band in the intensity spectrum) considering its codependency with the rest, but without quantifying time exchange between descriptors. A limitation of this analytical approach is that PLS regression models cannot be adjusted as usually done in linear regression. If covariates are included in the PLS model, they will contribute their shared variance with PA and the outcome. Aadland et al proposed obtaining residuals for the outcome from a linear regression model including confounders as explanatory variables, prior to entering the outcome variable in the PLS model. ${ }^{25} 3233$ This challenge remains for the analysis of categorical or time-to-event outcomes (eg, mortality).
Likewise, time-use behaviours or the intensity spectrum could be transformed as compositional data before performing multivariate pattern analysis. Since multivariate pattern analysis can handle singular data, the use of ILR coordinates is not necessary. Aadland et al recently compared the use of centred log ratio (CLR)-transformed time-use and intensity spectrum descriptors with respect to associations with metabolic health using multivariate pattern analysis. ${ }^{25}$ While associations appeared to differ, the interpretation of associations, considering the absolute and relative interpretation, were partly equivalent. The interpretation of CLR-transformed variables may not be very informative for public health messaging as they represent the effect of time exchange from the geometric mean of the time-use descriptors distribution to a specific time-use descriptor (eg, MVPA or any intensity spectrum band).

Other similar alternatives to reduce dimensionality of the data while retaining relevant information by increasing covariance among descriptors (rather than with the outcome) include factor analysis, principal component analysis, or joint and individual variation explained. ${ }^{34}$ This consensus group recommends considering these approaches to analyse many explanatory variables (eg, intensity spectrum) in relation to health. ${ }^{35}$ There is no clear recommendation on the number of bands (or number of explanatory variables) to generate for this analytical approach, though previous studies have used from 16 (uniaxial data) ${ }^{32}$ to 102 (triaxial data) ${ }^{33}$ intensity bands. Resolution (number of bands) may influence the relationship with the outcome and depend on the sample characteristics; thus, further research is needed.

\section{Intensity gradient and linear regression}

The intensity gradient describes the straight line negative slope of the natural logs of time and acceleration intensity. ${ }^{36}$ The intensity gradient was developed to: (1) capture the entire intensity distribution, (2) avoid reliance on population and protocolspecific calibration protocols, and (3) provide information that complements average acceleration. The intensity gradient can be used alongside average acceleration to more fully describe the 24-hour movement profile by capturing both volume and intensity of PA. Using the intensity gradient and average acceleration together in linear regression models allows investigation of independent, additive and interactive associations of volume and intensity of PA with health. More work is needed to interpret the intensity gradient relative to the adherence to PA guidelines.

\section{Intensity gradient or intensity distribution and functional data analysis}

The acceleration distribution over time of the day, the acceleration density or the intensity gradient function can be used in functional data analysis. Using scalar-on-function data analysis, ${ }^{37}$ these acceleration functions can be used as an explanatory variable in regression models including linear, ${ }^{38}$ logistic or Cox regression models. For example, in the case of the acceleration density function as explanatory variable, the association with the event of interest is described along the acceleration range. ${ }^{38}$ This shows acceleration sections that are associated with the outcome by accounting for the full acceleration distribution, allowing identification of a cut-point such that proportion of time spent above this acceleration cut-point is associated with the outcome. Once these cut-points are identified, it is possible to estimate differences in the outcome by allocating time below to time above this cut-point. ${ }^{39}$ 
Functional data analysis has several advantages: (1) it is not affected by multicollinearity since it handles the data continuity; (2) it can test the effects of time reallocation and thus consider closure; and (3) it detects sections of the accelerometer data that are important for a certain health outcome, thus relaxing assumptions of linearity in particular behaviours made by other statistical models. Among its main limitations, acceleration functions usually carry much information that may be irrelevant to the outcome, but is considered in the analysis. Its main drawback is difficulty translating the findings into useful, straightforward public health messages. Investigation of how to make the conclusions of functional data analysis relevant to public health guidelines is highly encouraged by this consensus group $\left(\mathrm{see}^{39}\right.$ for an example).

\section{MX metrics and linear regression}

MX metrics represent the acceleration above which a person's most active non-consecutive $\mathrm{X}$ minutes over the day are spent. An advantage of using MX metrics is that analysis is not affected by cut-point assumptions on energy expenditure, while cut-points may be post-hoc applied to enable public health messaging. For example, if the M60 of a child is $230 \mathrm{mg}$, this can be compared with an MVPA cut-point, for example, $200 \mathrm{mg}$,

${ }^{14}$ showing that the child meets the 60 min daily MVPA recommendation. However, if compared with a more stringent $250 \mathrm{mg}$ MVPA cut-point, the child does not reach the recommendation. The post-hoc application of cut-points can, therefore, be skipped and keep the interpretation to the descriptive MX values instead. Another advantage of this approach is that the intensity of PA for the specified duration is captured regardless of how inactive a person is. Regarding statistical modelling, the MX metrics usually include a wide range of variables (table 1, online supplemental appendix 1 (Section 1)). These MX metrics are likely to be codependent as they are time-use descriptors, which may increase the multicollinearity risk. Likewise, each MX metric would carry partial and relative information on the pattern, and compositional transformation would also be interesting, although this approach has not been tested yet.

The usefulness of MX metrics with multivariate pattern analysis has not been investigated yet. However, since one of the limitations of MX metrics with linear regression is collinearity among the explanatory variables, multivariate pattern analysis could provide new insights by overcoming collinearity.

\section{Multiple descriptors and machine learning}

Machine learning (ML) describes a broad range of techniques to automate finding patterns in data with a focus on predictive ability (supervised ML) or data reduction (unsupervised ML). ML methods have been widely applied to derive accelerometer descriptors, ${ }^{40}$ yet rarely applied to study health associations. ${ }^{41}{ }^{42}$ Different ML approaches have different strengths and limitations. In general, strengths include their usefulness for data-driven hypothesis generation, their capacity to handle multidimensional data, their ability to find non-linear patterns, and the possibility of training a model in one dataset and updating it in another. However, it can be difficult to interpret how results are obtained and their significance for public health guidelines. ML methods can also be data hungry and computationally intensive. Overfitting and sensitivity to (potentially unknown) biases in the training data are risks.

In some ways, multivariate pattern analyses and other dimension reduction methods can be considered ML methods. The Transparent Reporting of a multivariable prediction model for
Individual Prognosis Or Diagnosis (TRIPOD) Initiative developed a set of recommendations for reporting of studies developing, validating, or updating ML-based prediction models for diagnostic or prognostic purposes. ${ }^{43}$ The TRIPOD statement should be considered when developing or applying ML-based prediction models in physical behaviour epidemiology.

\section{FUTURE RESEARCH DIRECTIONS}

The workshop in Granada, as well as the later meeting and the work developed in the following months by this author group, has initiated a discussion on analytical approaches and their usefulness for public health guidelines. Currently, $150 \mathrm{~min} / \mathrm{week}$ of MVPA in adults and older adults, and $60 \mathrm{~min} / \mathrm{day}$ of MVPA in children are recommended by different agencies. ${ }^{22}{ }^{44}$ Recent guidelines removed the 10-minute bout requirement for MVPA in adults, and included the importance of replacing SB for PA. ${ }^{22}$ The Canadian PA guidelines are the first attempt to promote the combined effects of behaviours on health, ${ }^{45}$ although the evidence used was not based on the 24-hour paradigm (and so, appropriateness of statistical approach can be discussed).

We propose future research directions based on the research gaps identified, that is, the uncertainty regarding the accelerometer data descriptors to use and what analytical models are the most appropriate given the research question being addressed. The authors of this consensus article agree that investigations determining associations between physical behaviours and health should be extended to understand the interplay of physical behaviours (PA, SB and sleep) in their relationship with health. Measurement and processing capacity is increasing and offers an opportunity to provide further information on how different intensities and types of PA interact to improve health. At the same time, the focus on translating findings to meaningful information for interpretation in practice cannot be lost when using advanced analytical models. It is notable that most of the information presented comes from the PA and SB fields; thus, the relevance for the sleep research filed can be further discussed. The main implications for the analysis of accelerometer data proposed and agreed by the authors of this consensus manuscript are presented below.

\section{Short-term agenda}

- Clear communication on the rationale for the use and limitations of each analytical approach in studies is important for a meaningful interpretation of the findings. Practical recommendations for this are provided in the Analytical approaches section of this document and a decision tree was developed (figure 1) to assist researchers' decision-making.

- Investigation of the associations of physical behaviours with health using different analytical approaches is encouraged. Ideally, physical behaviour epidemiology would draw consistent conclusions independently of analytical approach. To do so, clear reporting on the interpretation of findings derived from each analytical approach is crucial to understand 'a priori' inconsistencies across methods. Triangulation of results from different analytical approaches is currently the best solution to quantify associations of physical behaviours with health. Additionally, using the best-suited analytical approaches for a given research question is crucial (see figure 1).

- Although little explored so far, ML-based approaches for diagnostic/prognostic purposes are worth implementing. We encourage transparent reporting of the resulting tools (TRIPOD initiative checklist). 
Raw accelerometer data

(this applies to data from hip-, wrist-, thigh-worn accelerometers or any other attachment site)

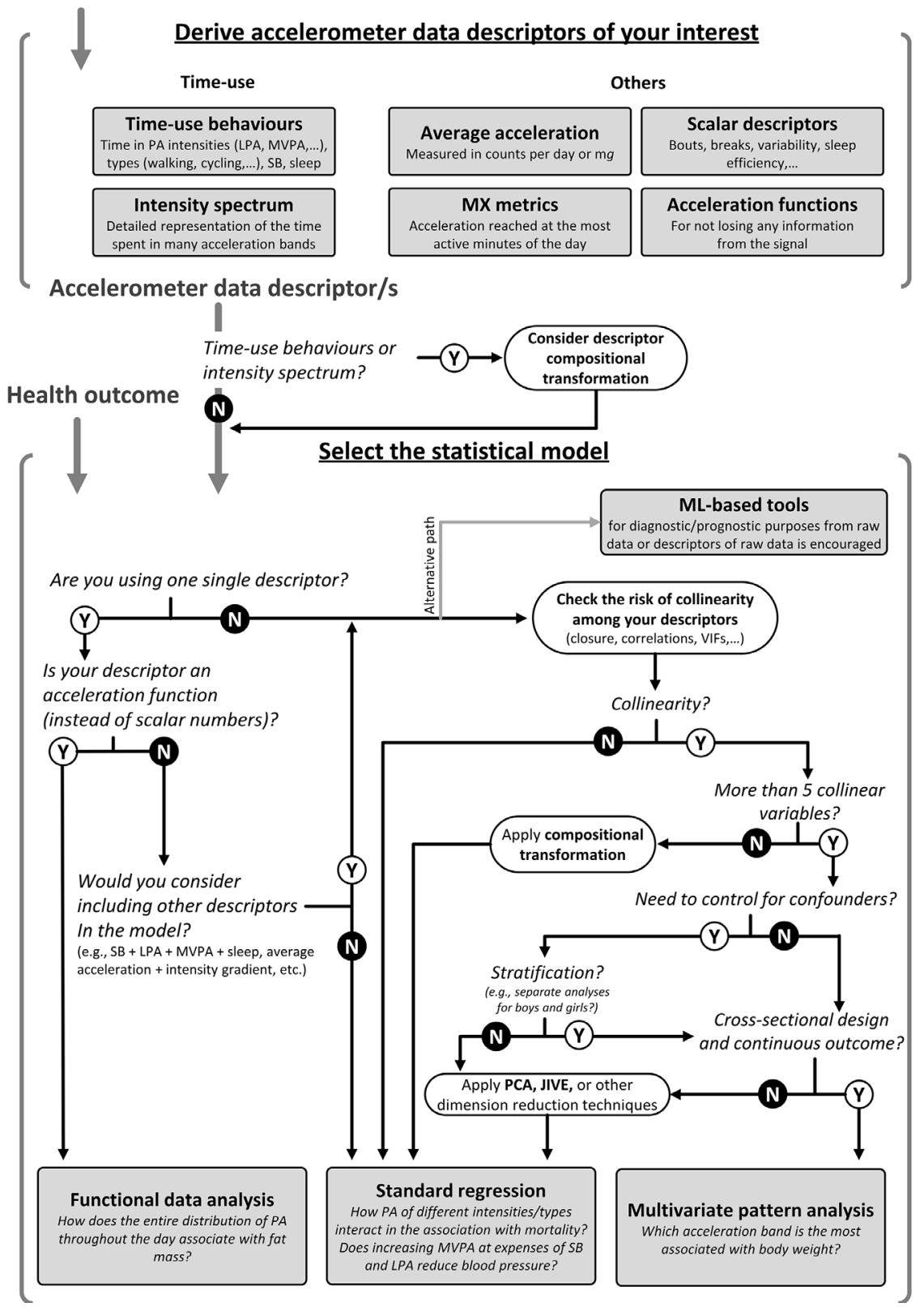

Figure 1 The GRANADA consensus decision tree and research question examples to assist in the selection of an analytical approach in the field of 'physical behaviour epidemiology'. JIVE, joint and individual variance explained; LPA, light physical activity; ML, machine learning; MVPA, moderateto-vigorous physical activity; PA, physical activity; PCA, principal component analysis; SB, sedentary behaviour; VIFs, variance inflation factors.

- Translating findings to meaningful information for guidelines should be a priority. Accurate reporting of study findings, interpretation and practical implications is highly encouraged.

\section{Long-term agenda}

- How to conveniently adjust for confounders in multivariate pattern analysis should be investigated and its application extended to time-dependent outcomes (eg, survival analysis with mortality).

- Further efforts are needed to translate functional data analysis and other advanced analytical approaches' outputs into meaningful information for public health guidelines.
- To evaluate whether the information gathered from the analytical approaches discussed herein can result in complementary information for public health guidelines. Such complementary information may result in more specific recommendations for certain health outcomes or populations, or even in their implementation at population level through movement sensors using evidence-based goals on PA intensity, duration, timing or type, among others.

\section{CONCLUSIONS}

This group agreed on several consensus points and research needs for physical behaviour epidemiology (see box 1 and figure 1). This consensus article will increase researchers' understanding 
Box 1 Consensus points from the GRANADA report on analytical approaches to assess associations with accelerometer-determined physical behaviours (physical activity (PA), sedentary behaviour (SB) and sleep) in epidemiological studies

1. The study of the association between physical behaviours (ie, PA, SB and sleep) and health should move to a more thorough investigation of the interactions and codependencies between different behaviours (or PA intensities) and health. Several analytical approaches are provided in this consensus document, although none of them is free from limitations.

2. We recommend investigating more detailed PA intensities than the typically studied (ie, SB and moderate-to-vigorous PA). Examples include light PA of different intensities or the more fine-grained intensity bands as described in this document.

3. Public health guidelines on physical behaviours should acknowledge that behaviours are codependent and this may affect the guidelines as traditionally understood.

4. Further investigation in functional data analysis and machine learning is needed concerning the associations of physical behaviours with health.

5. There is not a gold standard able to test which analytical approach is the best for a given research question. Thus, we cannot make a strong recommendation on a single analytical approach. Instead, we provide some practical recommendations to select analytical approaches well suited for a given research question. Triangulation across findings from different analytical approaches is currently the best solution.

of different analytical approaches used in recent epidemiological studies of physical behaviours. This article and the decision tree provided aim to assist researchers in selecting analytical approaches based on their research questions and data. This will ultimately have an impact on the scientific evidence and, therefore, on future public health guidelines on physical behaviours.

\section{Author affiliations}

"PROFITH "PROmoting FITness and Health through physical activity" Research Group, Sport and Health University Research Institute (iMUDS), Department of Physical Education and Sports, Faculty of Sport Sciences, University of Granada, Granada, Spain

${ }^{2}$ Department of Health, Medicine and Caring Sciences, Linköping University, Linköping, Sweden

${ }^{3}$ Faculty of Education, Arts and Sports, Western Norway University of Applied Sciences, Sogndal, Norway

${ }^{4}$ Department of Sport Science and Biomechanics, University of Southern Denmark, Odense, Denmark

${ }^{5}$ School of Health and Life Science, Glasgow Caledonian University, Glasgow, UK ${ }^{6}$ Department of Movement and Sport Science, Ghent University, Ghent, Belgium ${ }^{7}$ Department of Sports Medicine, Norwegian School of Sport Sciences, Osloål, Norway ${ }^{8}$ Departement of Sport Science and Physical Education, University of Agder, Kristiansand, Norway

${ }^{9}$ Department of Chronic Diseases, National Institute for Health Development, Tallinn, Estonia

${ }^{10}$ School of Natural Sciences and Health, Tallinn University, Tallinn, Estonia ${ }^{11}$ Institute of Psychology, University of Tartu, Tartu, Estonia

${ }^{12}$ Department of Chemistry, University of Bergen, Bergen, Norway

${ }^{13}$ Biomathematics and Statistics Scotland, Edinburgh, UK

${ }^{14}$ Diabetes Research Centre, University of Leicester, Leicester General Hospital, Leicester, UK

${ }^{15} \mathrm{NIHR}$ Leicester Biomedical Research Centre, Leicester General Hospital, Leicester, UK
${ }^{16}$ Alliance for Research in Exercise, Nutrition and Activity (ARENA), Sansom Institute for Health Research, Division of Health Sciences, University of South Australia, Adelaide, SA, Australia

${ }^{17}$ Université de Paris, Inserm U1153, Epidemiology of Ageing and Neurodegenerative diseases, Paris, France

${ }^{18}$ Department of Epidemiology and Public Health, University College London, London, UK

${ }^{19}$ Accelting, Almere, The Netherlands

${ }^{20}$ Amsterdam UMC, Vrije Universiteit Amsterdam, Department of Public and Occupational Health, Amsterdam Public Health research institute, Amsterdam, The Netherlands

${ }^{21}$ Nuffield Department of Population Health, University of Oxford, Oxford, UK

${ }^{22}$ Big Data Institute, Li Ka Shing Centre for Health Information and Discovery, University of Oxford, Oxford, UK

${ }^{23}$ Department of Biosciences and Nutrition, Karolinska Institutet, Huddinge, Sweden

Correction notice This article has been corrected since it published Online First. The co-corresponding author has been added.

Twitter Jairo H Migueles@Jairohm8 and Séverine Sabia @epiAgeing

Acknowledgements Because of logistic and funding reasons, the researchers participating in the workshop and authors of this manuscript are mainly based in Europe and do not represent governing body or public health organisation. Thus, the authors of this consensus manuscript are a group of European researchers selected from the speaker and participant list of the GRANADA workshop: 'a focus on statistical methods to analyse accelerometer-measured physical activity'. Additionally, the manuscript was sent for review to a set of external experts in the field from different countries, including Europe, USA and Australia. Ten reviewers provided comments (more than 150 comments received) out of the 15 initially contacted. Therefore, it has an international dimension beyond Europe given the important input that the comments from the international review group had on the manuscript. The authors jointly accumulate reasonable expertise around methodology and application of methodology in physical behaviour research. This manuscript contains the authors' opinions and it is not written under the name of any health association or governing body. This is part of a PhD Thesis conducted in the Biomedicine Doctoral Studies at the University of Granada, Spain.

Collaborators Alexander Burchartz (Institute for Sports and Sports Science, Karlsruhe Institute of Technology, Karlsruhe, Germany), Cain Clark (Centre for Sport, Exercise and Life Sciences, Coventry University, Coventry, UK), Paddy Dempsey (MRC Epidemiology Unit, Institute of Metabolic Science, University of Cambridge, Cambridge, UK; Physical Activity \& Behavioural Epidemiology Laboratories, Baker Heart and Diabetes Institute, Melbourne, Australia), Aiden Doherty (Nuffield Department of Population Health, Big Data Institute, University of Oxford, Oxford, UK; NIHR Oxford Biomedical Research Centre, Oxford University Hospitals NHS Foundation Trust, John Radcliffe Hospital, Oxford, UK), Ulf Ekelund (Department of Sports Medicine, Norwegian School of Sport Sciences, Oslo, Norway), Timothy Olds (Alliance for Research in Exercise, Nutrition and Activity, University of South Australia, Adelaide, Australia), Eric J Shiroma (Laboratory of Epidemiology and Population Science, National Institute on Aging, Baltimore, Maryland), Emmanuel Stamatakis (Charles Perkins Centre, Prevention Research Collaboration, Sydney School of Public Health, Faculty of Medicine and Health, University of Sydney, Sydney, NSW, Australia), Richard P Troiano (Division of Cancer Control and Population Sciences, National Cancer Institute, NIH, HHS, Rockville, Maryland, USA), Stewart Trost (Institute of Health and Biomedical Innovation at Queensland Centre for Children's Health Research, Queensland University of Technology, South Brisbane, Australia; Faculty of Health, School of Exercise and Nutrition Sciences, Queensland University of Technology, Brisbane, Australia) and Vadim Zipunnikov (Department of Biostatistics, Bloomberg School of Public Health, Johns Hopkins University).

Contributors JHM and FBO conceived the idea, organised and led the workshop, the meeting, and the GRANADA consensus design and writing. All authors contributed to the idea, reviewed the manuscript and provided detailed suggestions for revisions.

Funding This study was conducted under the umbrella of the ActiveBrains and the SmarterMove projects supported by the MINECO/FEDER (DEP2013-47540, DEP2016-79512-R, RYC-2011-09011) and the CoCA project supported by the European Union's 2020 research and innovation programme (667302). JHM is supported by a grant from the Spanish Ministry of Education, Culture and Sport (FPU15/02645). AR is supported by the NIHR Leicester Biomedical Research Centre, and the Collaboration for leadership in Applied Health Research and Care (CLAHRC) East Midlands. SS is supported by the French National Research Agency (ANR-19CE36-0004-01). RW is supported by a Medical Research Council Industrial Strategy Studentship (MR/S502509/1). Additional funding was obtained from the Andalusian Operational Programme supported with European Regional Development Funds (ERDF in English, FEDER in Spanish, project ref: B-CTS-355-UGR18), the University of Granada, Plan Propio de Investigación 2016, Excellence actions: Units of Excellence; Scientific Excellence Unit on Exercise and Health (UCEES), Junta de Andalucía, 
Consejería de Conocimiento, Investigación y Universidades and European Regiona Development Funds (ref. SOMM17/6107/UGR). In addition, funding was provided by the SAMID III network, RETICS, funded by the PN I+D+I 2017-2021 (Spain), ISCIIISub-Directorate General for Research Assessment and Promotion, the European Regional Development Fund (ERDF) (Ref. RD16/0022), the EXERNET Research Network on Exercise and Health in Special Populations (DEP2005-00046/ACTI).

\section{Competing interests None declared.}

Patient consent for publication Not required.

Provenance and peer review Not commissioned; externally peer reviewed.

Supplemental material This content has been supplied by the author(s). It has not been vetted by BMJ Publishing Group Limited (BMJ) and may not have been peer-reviewed. Any opinions or recommendations discussed are solely those of the author(s) and are not endorsed by BMJ. BMJ disclaims all liability and responsibility arising from any reliance placed on the content. Where the content includes any translated material, BMJ does not warrant the accuracy and reliability of the translations (including but not limited to local regulations, clinical guidelines, terminology, drug names and drug dosages), and is not responsible for any error and/or omissions arising from translation and adaptation or otherwise.

Open access This is an open access article distributed in accordance with the Creative Commons Attribution Non Commercial (CC BY-NC 4.0) license, which permits others to distribute, remix, adapt, build upon this work non-commercially, and license their derivative works on different terms, provided the original work is properly cited, appropriate credit is given, any changes made indicated, and the use is non-commercial. See: http://creativecommons.org/licenses/by-nc/4.0/.

\section{ORCID iDs}

Jairo H Migueles http://orcid.org/0000-0003-0366-6935

Eivind Aadland http://orcid.org/0000-0001-9654-2653

Lars Bo Andersen http://orcid.org/0000-0002-9800-029X

Jan Christian Brønd http://orcid.org/0000-0001-6718-3022

Sebastien F Chastin http://orcid.org/0000-0003-1421-9348

Bjørge H Hansen http://orcid.org/0000-0001-8445-8227

Kenn Konstabel http://orcid.org/0000-0002-9673-8469

Olav Martin Kvalheim http://orcid.org/0000-0001-9432-8776

Duncan E McGregor http://orcid.org/0000-0003-2575-7547

Alex V Rowlands http://orcid.org/0000-0002-1463-697X

Séverine Sabia http://orcid.org/0000-0003-3109-9720

Vincent T van Hees http://orcid.org/0000-0003-0182-9008

Rosemary Walmsley http://orcid.org/0000-0002-1916-2478

Francisco B Ortega http://orcid.org/0000-0003-2001-1121

\section{REFERENCES}

1 Bussmann JBJ, van den Berg-Emons RJG. To total amount of activity... and beyond: perspectives on measuring physical behavior. Front Psychol 2013;4:463.

2 Ekelund U, Steene-Johannessen J, Brown WJ, et al. Does physical activity attenuate, or even eliminate, the detrimental association of sitting time with mortality? A harmonised meta-analysis of data from more than 1 million men and women. Lancet 2016;388:1302-10.

3 Stamatakis E, Gale J, Bauman A, et al. Sitting time, physical activity, and risk of mortality in adults. J Am Coll Cardiol 2019;73:2062-72.

4 Migueles JH, Cadenas-Sanchez C, Rowlands AV, et al. Comparability of accelerometer signal aggregation metrics across placements and dominant wrist cut points for the assessment of physical activity in adults. Sci Rep 2019;9:18235.

5 Migueles JH, Cadenas-Sanchez C, Ekelund U, et al. Accelerometer data collection and processing criteria to assess physical activity and other outcomes: a systematic review and practical considerations. Sports Med 2017;47:1821-45.

6 Kamada M, Shiroma EJ, Harris TB, et al. Comparison of physical activity assessed using hip- and wrist-worn accelerometers. Gait Posture 2016:44:23-8.

7 LaMunion SR, Bassett DR, Toth LP, et al. The effect of body placement site on ActiGraph wGT3X-BT activity counts. Biomed. Phys. Eng. Express 2017;3:035026.

8 Hickey AM, Freedson PS. Utility of consumer physical activity Trackers as an intervention tool in cardiovascular disease prevention and treatment. Prog Cardiovasc Dis 2016:58:613-9.

9 Togo $\mathrm{F}$, Watanabe E, Park $\mathrm{H}$, et al. How many days of pedometer use predict the annual activity of the elderly reliably? Med Sci Sports Exerc 2008;40:1058-64.

10 Ortega FB, Konstabel K, Pasquali E, et al. Objectively measured physical activity and sedentary time during childhood, adolescence and young adulthood: a cohort study. PLoS One 2013;8:e60871.

11 Konstabel K, Veidebaum T, Verbestel V, et al. Objectively measured physical activity in European children: the IDEFICS study. Int J Obes 2014;38:S135-43.

12 Rääsk T, Lätt E, Jürimäe T, et al. Association of subjective ratings to objectively assessed physical activity in pubertal boys with differing BMI. Percept Mot Skills 2015;121:245-59.

13 Evenson KR, Catellier DJ, Gill K, et al. Calibration of two objective measures of physical activity for children. J Sports Sci 2008;26:1557-65.
14 Hildebrand M, VAN Hees VT, Hansen BH, et al. Age group comparability of raw accelerometer output from wrist- and hip-worn monitors. Med Sci Sports Exerc 2014;46:1816-24

15 Chandler JL, Brazendale K, Beets MW, et al. Classification of physical activity intensities using a wrist-worn accelerometer in 8-12-year-old children. Pediatr Obes 2016;11:120-7.

16 Crowley P, Skotte J, Stamatakis E, et al. Comparison of physical behavior estimates from three different thigh-worn accelerometers brands: a proof-of-concept for the prospective physical activity, sitting, and sleep Consortium (ProPASS). Int I Behav Nutr Phys Act 2019;16:65.

17 Sadeh A, Sharkey KM, Carskadon MA. Activity-based sleep-wake identification: an empirical test of methodological issues. Sleep 1994;17:201-7.

18 van Hees VT, Sabia S, Anderson KN, et al. A novel, open access method to assess sleep duration using a wrist-worn accelerometer. PLoS One 2015;10:e0142533-13.

19 Burchartz A, Anedda B, Auerswald T, et al. Assessing physical behavior through accelerometry - state of the science, best practices and future directions. Psychol Sport Exerc 2020;49:101703.

20 Rowlands A, Davies M, Dempsey P, et al. Wrist-worn accelerometers: recommending $\sim 1.0 \mathrm{mg}$ as the minimum clinically important difference (MCID) in daily average acceleration for inactive adults. Br J Sports Med 2020:bjsports-2020-102293.

21 Jakicic JM, Kraus WE, Powell KE, et al. Association between bout duration of physical activity and health: systematic review. Med Sci Sports Exerc 2019;51:1213-9.

22 Bull FC, Al-Ansari SS, Biddle S, et al. World Health organization 2020 guidelines on physical activity and sedentary behaviour. Br I Sports Med 2020;54:1451-62.

23 Stanton JM. Galton, Pearson, and the peas: a brief history of linear regression for statistics Instructors. J Stat Educ 2001;9.

24 Dumuid D, Stanford TE, Martin-Fernández J-A, et al. Compositional data analysis for physical activity, sedentary time and sleep research. Stat Methods Med Res 2018;27:3726-38.

25 Aadland E, Kvalheim OM, Anderssen SA, et al. Multicollinear physical activity accelerometry data and associations to cardiometabolic health: challenges, pitfalls, and potential solutions. Int J Behav Nutr Phys Act 2019:16:1-14.

26 Chastin SFM, Palarea-Albaladejo J, Dontje ML, et al. Combined effects of time spent in physical activity, sedentary behaviors and sleep on obesity and cardiometabolic health markers: a novel compositional data analysis approach. PLoS One 2015;10:e0139984

27 Ekelund U, Tarp J, Steene-Johannessen J, et al. Dose-response associations between accelerometry measured physical activity and sedentary time and all cause mortality: systematic review and harmonised meta-analysis. BMJ 2019;366:14570

28 Tarp J, Hansen BH, Fagerland MW, et al. Accelerometer-measured physical activity and sedentary time in a cohort of US adults followed for up to 13 years: the influence of removing early follow-up on associations with mortality. Int I Behav Nutr Phys Act 2020:17:1-8.

29 Lee I-M, Shiroma EJ, Evenson KR, et al. Accelerometer-measured physical activity and sedentary behavior in relation to all-cause mortality: the women's health study. Circulation 2018;137:203-5.

30 McGregor DE, Palarea-Albaladejo J, Dall PM, et al. Compositional analysis of the association between mortality and 24-hour movement behaviour from NHANES. Eur $\int$ Prev Cardiol 2019:204748731986778.

31 Biddle GJH, Edwardson CL, Henson J, et al. Associations of physical behaviours and behavioural Reallocations with markers of metabolic health: a compositional data analysis. Int J Environ Res Public Health 2018;15:2280-14.

32 Aadland E, Kvalheim OM, Anderssen SA, et al. The multivariate physical activity signature associated with metabolic health in children. Int J Behav Nutr Phys Act 2018;15:1-11

33 Aadland E, Kvalheim OM, Anderssen SA, et al. The Triaxial physical activity signature associated with metabolic health in children. Med Sci Sports Exerc 2019;51:2173-9.

34 Di J, Spira A, Bai J, et al. Joint and individual representation of domains of physical activity, sleep, and circadian rhythmicity. Stat Biosci 2019;11:371-402.

35 Aadland E, Nilsen AKO, Andersen LB, et al. A comparison of analytical approaches to investigate associations for accelerometry-derived physical activity spectra with health and developmental outcomes in children. J Sports Sci 2021;39:1-9.

36 Rowlands AV, Edwardson CL, Davies MJ, et al. Beyond cut points: Accelerometer metrics that capture the physical activity profile. Med Sci Sports Exerc 2018:50:1323-32.

37 Ramsay J, Silverman B. Functional data analysis. 2 edn. New York, 2005.

38 Augustin NH, Mattocks C, Faraway JJ, et al. Modelling a response as a function of high-frequency count data: the association between physical activity and fat mass. Stat Methods Med Res 2017:26:2210-26.

39 Benadjaoud MA, Menai M, van Hees VT, et al. The association between accelerometerassessed physical activity and respiratory function in older adults differs between smokers and non-smokers. Sci Rep 2019;9:1-9.

40 Narayanan A, Desai F, Stewart T, et al. Application of raw Accelerometer data and Machine-Learning techniques to characterize human movement behavior: a systematic scoping review. J Phys Act Health 2020;17:360-83.

41 Hua A, Quicksall Z, Di C, et al. Accelerometer-based predictive models of fall risk in older women: a pilot study. NPJ Digit Med 2018;1:25 
$42 \mathrm{Li}$, Zhao H. Automated feature extraction from population wearable device data identified novel loci associated with sleep and circadian rhythms. PLoS Genet 2020;16:e1009089.

43 Collins GS, Moons KGM. Reporting of artificial intelligence prediction models. Lancet 2019:393:1577-9.

44 U.S. Department of Health and Human Services. 2018 physical activity guidelines Advisory Committee scientific report. Washington, DC. Available: https://health. gov/paguidelines/second-edition/report/pdf/pag_advisory_committee_report.pdf\% OAhttps://health.gov/paguidelines/second-edition/report/pdf/06_E_Systematic_ Review_Literature_Search_Methodology.pdf

45 Canadian Society for Exercise Physiology. Canadian physical activity guidelines: clinical practice Guideline development report, 2011.

46 Fairclough SJ, Taylor S, Rowlands AV, et al. Average acceleration and intensity gradient of primary school children and associations with indicators of health and well-being. $J$ Sports Sci 2019:37:2159-67.

47 Lee I-M, Shiroma EJ, Kamada M, et al. Association of step volume and intensity with all-cause mortality in older women. JAMA Intern Med 2019;02215. doi:10.1001/ jamainternmed.2019.0899. [Epub ahead of print: 29 May 2019].

48 Saint-Maurice PF, Troiano RP, Bassett DR, et al. Association of daily step count and step intensity with mortality among US adults. JAMA 2020;323:1151-60.

49 Mora-Gonzalez J, Esteban-Cornejo I, Cadenas-Sanchez C, et al. Fitness, physical activity, working memory, and neuroelectric activity in children with overweight/ obesity. Scand J Med Sci Sports 2019;29:1352-63.

50 Migueles JH, Cadenas-Sanchez C, Tudor-Locke C, et al. Comparability of published cut-points for the assessment of physical activity: implications for data harmonization. Scand J Med Sci Sports 2019;29:566-74.

51 Tudor-Locke C, Aguiar EJ, Han H, et al. Walking cadence (steps/min) and intensity in 21-40 year olds: CADENCE-adults. Int J Behav Nutr Phys Act 2019:16:8

52 Tudor-Locke C, Schuna JM, Han H, et al. Cadence (steps/min) and intensity during ambulation in 6-20 year olds: the CADENCE-kids study. Int J Behav Nutr Phys Act 2018;15:20.

53 Barreira TV, Katzmarzyk PT, Johnson WD, et al. Cadence patterns and peak cadence in US children and adolescents: NHANES, 2005-2006. Med Sci Sports Exerc 2012;44:1721-7.

54 Rowlands AV, Dawkins NP, Maylor B, et al. Enhancing the value of accelerometerassessed physical activity: meaningful visual comparisons of data-driven translational accelerometer metrics. Sports Med Open 2019;5:47.

55 Tudor-Locke C, Brashear MM, Katzmarzyk PT, et al. Peak stepping cadence in freeliving adults: 2005-2006 NHANES. J Phys Act Health 2012:9:1125-9.

56 Augustin NH, Mattocks C, Cooper AR, et al. Modelling fat mass as a function of Weekly physical activity profiles measured by actigraph accelerometers. Physiol Meas 2012:33:1831-9.
57 Wanigatunga AA, Di J, Zipunnikov V, et al. Association of total daily physical activity and fragmented physical activity with mortality in older adults. JAMA Netw Open 2019;2:e1912352.

58 Kok JS, Berg IJ, Blankevoort GCG, et al. Rest-activity rhythms in small scale homelike care and traditional care for residents with dementia. BMC Geriatr 2017;17:137.

59 Henson J, Yates T, Biddle SJH, et al. Associations of objectively measured sedentary behaviour and physical activity with markers of cardiometabolic health. Diabetologia 2013;56:1012-20.

60 Mora-Gonzalez J, Esteban-Cornejo I, Cadenas-Sanchez C, et al. Physical fitness, physical activity, and the executive function in children with overweight and obesity. $J$ Pediatr 2019;208:50-6.

61 Grgic J, Dumuid D, Bengoechea EG, et al. Health outcomes associated with reallocations of time between sleep, sedentary behaviour, and physical activity: a systematic scoping review of isotemporal substitution studies. Int I Behav Nutr Phys Act 2018:15:1-68.

62 Mekary RA, Willett WC, Hu FB, et al. Isotemporal substitution paradigm for physical activity epidemiology and weight change. Am J Epidemiol 2009;170:519-27.

63 Kvalheim OM, Arneberg R, Grung B. Determination of optimum number of components in partial least squares regression from distributions of the root-mean-squared error obtained by Monte Carlo resampling. J Chemom 2018:32:e2993-12.

64 Rajalahti T, Arneberg R, Berven FS, et al. Biomarker discovery in mass spectral profiles by means of selectivity ratio plot. Chemom Intell Lab Syst 2009;95:35-48.

65 Rajalahti T, Kvalheim OM. Multivariate data analysis in Pharmaceutics: a tutorial review. Int J Pharm 2011;417:280-90.

66 Aadland E, Andersen LB, Resaland GK, et al. Interpretation of multivariate association patterns between Multicollinear physical activity Accelerometry data and cardiometabolic health in Children-A tutorial. Metabolites 2019:9:129-14.

67 Menai M, van Hees VT, Elbaz A, et al. Accelerometer assessed moderate-to-vigorous physical activity and successful ageing: results from the Whitehall II study. Sci Rep 2017:8:1-9

68 Goldsmith J, Zipunnikov V, Schrack J. Generalized multilevel function-on-scalar regression and principal component analysis. Biometrics 2015;71:344-53.

69 Sørensen H, Goldsmith J, Sangalli LM. An introduction with medical applications to functional data analysis. Stat Med 2013;32:5222-40.

$70 \mathrm{Bi} \mathrm{Q}$, Goodman KE, Kaminsky J, et al. What is machine learning? A primer for the epidemiologist. Am J Epidemiol;3.

71 Alaa AM, Bolton T, Di Angelantonio E, Di AE, et al. Cardiovascular disease risk prediction using automated machine learning: a prospective study of 423,604 UK Biobank participants. PLoS One 2019:14:e0213653-17. 